Article

\title{
Bio-Refining of Carbohydrate-Rich Food Waste for Biofuels
}

\section{Hoang-Tuong Nguyen Hao ${ }^{1}$, Obulisamy Parthiba Karthikeyan ${ }^{1,2}$ and Kirsten Heimann 1,2,3,4,*}

1 College of Marine and Environmental Sciences, James Cook University, Townsville 4811, Queensland, Australia

2 Comparative Genomics Centre, James Cook University, Townsville 4811, Queensland, Australia

3 Centre for Bio-discovery and Molecular Development of Therapeutics, James Cook University, Townsville 4811, Queensland, Australia

4 Centre for Sustainable Tropical Fisheries and Aquaculture, James Cook University, Townsville 4811, Queensland, Australia

* Author to whom correspondence should be addressed; E-Mail: kirsten.heimann@jcu.edu.au; Tel.: +61-07-4781-5795; Fax: +61-07-4725-1570.

Academic Editor: Clifford Louime

Received: 1 March 2015 / Accepted: 9 June 2015 / Published: 25 June 2015

Abstract: The global dependence on finite fossil fuel-derived energy is of serious concern given the predicted population increase. Over the past decades, bio-refining of woody biomass has received much attention, but data on food waste refining are sorely lacking, despite annual and global deposition of 1.3 billion tons in landfills. In addition to negative environmental impacts, this represents a squandering of valuable energy, water and nutrient resources. The potential of carbohydrate-rich food waste (CRFW) for biofuel (by Rhodotorulla glutinis fermentation) and biogas production (by calculating theoretical methane yield) was therefore investigated using a novel integrated bio-refinery approach. In this approach, hydrolyzed CRFW from three different conditions was used for Rhodotorulla glutinis cultivation to produce biolipids, whilst residual solids after hydrolysis were characterized for methane recovery potential via anaerobic digestion. Initially, CRFW was hydrolysed using thermal- (Th), chemical- (Ch) and Th-Ch combined hydrolysis (TCh), with the CRFW-leachate serving as a control (Pcon). Excessive foaming led to the loss of TCh cultures, while day-7 biomass yields were similar (3.4-3.6 g dry weight (DW) $\mathrm{L}^{-1}$ ) for the remaining treatments. Total fatty acid methyl ester (FAME) content of $R$. glutinis cultivated on CRFW hydrolysates were relatively low $(\sim 6.5 \%)$ but quality parameters (i.e., cetane number, density, viscosity and higher heating values) of 
biomass extracted biodiesel complied with ASTM standards. Despite low theoretical RS-derived methane potential, further research under optimised and scaled conditions will reveal the potential of this approach for the bio-refining of CRFW for energy recovery and value-added co-product production.

Keywords: anaerobic digestion; biodiesel; fatty acid methyl ester (FAME); fermentation; Rhodotorula glutinis; yeast

\section{Introduction}

Bio-refining is an alternative to fossil fuel-based refining yielding high-value, low-volume, marketable products, i.e., polymers, pigments, nutraceuticals and biofuels as co-products [1,2]. It is defined as a more sustainable, efficient and flexible process for complete conversion of biomass into value-added products through integrated approaches [3]. Conventionally a variety of processes such as fractiontionation, liquefaction, hydrolysis, fermentation, pyrolysis, hydrothermal carbonization and bio-gasification are included to recycle waste biomass into value-adding products [2]. Employed refining processes are grouped into four main categories based on energy, economic and by-product developments, i.e., gasification-, pyrolysis-, hydrothermal- and fermentation-based bio-refineries $[1,2,4]$. A wide range of feedstocks can be bio-refined, but the selection of appropriate processes and their integration is important, yet little information is available at this stage for effective strategies for the bio-refining of food wastes, i.e., more than $80 \%$ biomass feedstock used in bio-refineries are wood and shrubs $[2,5]$. Technically feasible separation operation of the biomass, which would enable separate use or subsequent processing of whole biomass as feedstock through integrated biorefinery approaches are still in initial stages of investigation [6].

Globally, $\sim 1.3$ billion metric tons of food waste is generated annually which is estimated to increase in parallel with population growth [7,8]. Within the Australian context, the disposal of 2.29 million tons of food annually to landfills and the resulting biogenic degradation leads to emission of 11 million tons of greenhouse gas emissions (as $\mathrm{CO}_{2}$ (g) equivalents; [9]). The current technological capability offers few options for the transformation of food waste into bio-energy, but the net energy yields often exceed total energy input (e.g., pyrolysis-based bio-refining exceeded by 20\%; [10]). Fermentation-based bio-refining (i.e., anaerobic digestion (AD), composting and direct fermentation processes) are widely accepted methods for food waste treatment yielding favourable end products such as biogas, bio-fertilizers and industrially important acids and alcohols (i.e., lactic acid, succinic acid, acetic acid, ethanol, methanol, etc.) requiring low energy inputs [11-13]. The main drawbacks with these conventional technologies are time efficiency (21 and 60 days for $\mathrm{AD}$ and composting, respectively), foot print area requirements, $\mathrm{GHG}$ emissions (i.e., $\mathrm{CO}_{2}$ and ammonia during composting), and process-associated health risks due to pathogen spread/odours (i.e., composting). Fermentation-based approaches are widely used either on their own or in combination with other treatment technologies for bio-refining of food waste, mainly to: (i) maximize recycling of nutrients/energy; and (ii) reduce treatment cost, time requirements and environmental burdens. However, there is no appropriate technology integration for mixed food waste treatment coupling energy and nutrient recovery potentials. 
Mixed food wastes are rich in sugars, proteins, lipids, vitamins and minerals which are easily assimilated as raw materials for the production of high-value chemicals, bio-oil and polymers by microorganisms such as bacteria, yeast, fungi or algae $[8,14]$. Yeast is considered superior over other microorganisms due to high biomass growth rates, lipid accumulation capacity, fatty acid profile, and carotenoid, biopolymer and nutraceutical potential $[15,16]$. Although there are 600 identified yeast species, fewer than 30 species can be categorised as oleaginous with the lipidome accounting for $>20 \%$ biomass and only very few produce carotenoids and biopolymers [17,18]. In addition, data on bio-refining of carbohydrate-rich food waste using yeast are limited, i.e., largely confined to reported yeast biomass production on other media. For instance, Yerrowia lipolytica (phylum Ascomycota) has been used for bio-refining of lipid-rich restaurant-food waste, which proved to be an excellent cultivation medium when coupled with hydrolysis of the complex sugars [19]. In contrast, the red yeast Rhodotorula glutinis (phylum Basidiomycota) has been cultivated on different wastewater media [16], food waste and municipal wastewater mixed media [20], glycerol- [15] and starch-containing wastewaters [21]. Another yeast, Cryptococcus curvatus showed higher productivity than Y. lipolytica when cultured on glucose-based media, but lipid content was $10 \%$ lower than $R$. glutinis cultured on food waste hydrolysates [20]. More than $70 \%$ increase in lipidome content per gram dry biomass has been achieved in R. glutinis under nutrient-limited (nitrogen and phosphate) culture conditions [22,23]. Of the total lipidome accumulated and synthesized, $\geq 90 \%$ is comprised solely of triacylglycerides (TAG), free fatty acids $(<1 \%)$ (FFA) and steryl esters (SE) [24]. Furthermore, $R$. glutinis accumulate the high value bio-product $\beta$-carotene ( $1 \mathrm{~kg} \sim 1,600 \mathrm{USD})$ used commercially as a food colorant, nutritional supplement, cosmetic colorant, antioxidant and anticancer agent in pharmaceuticals [16].

These industrially relevant characteristics of $R$. glutinis make it a potential candidate for developing a fermentative bio-refinery concept for food waste treatment. As such, this study aimed to investigate an integrated bio-refinery process consisting of hydrolysis of carbohydrate-rich food waste (CRFW) and yeast fermentation of the resulting hydrolysate using $R$. glutinis for lipid accumulation. The residual solid from the hydrolysis pre-treatment were characterized for bioenergy potential to maximize energy recycling in the proposed bio-refinery approach (Figure 1). Effects of three different hydrolytic pre-treatments (thermal, chemical and thermo-chemical) on fermentative biolipid production and bioenergy recovery potential during the fermentative bio-refinery process of CRFW were investigated and results are discussed.

\section{Materials and Methods}

\subsection{R. glutinis Culturing and Acclimatization}

A pure culture of $R$. glutinis FRR-4522, an isolate from dairy produce was supplied by the Commonwealth Scientific and Industrial Research Organisation (CSIRO), Australia. R. glutinis was subcultured and maintained on $2 \%$ agar plates prepared with Yeast Malt (YM) medium ( $3 \mathrm{~g} \mathrm{~L}^{-1}$ yeast extract; $3 \mathrm{~g} \mathrm{~L}^{-1}$ malt extract; $5 \mathrm{~g} \mathrm{~L}^{-1}$ casein peptone; $10 \mathrm{~g} \mathrm{~L}^{-1}$ dextrose). Cultivation of $R$. glutinis for CRFW conversion consisted of $350 \mathrm{~mL}$ YM broth and $100 \mathrm{~mL}$ hydrolysed CRFW (hydrolysis procedures are detailed in section 2.2) at a pH of $4 \pm 0.01$ in $1 \mathrm{~L}$ Erlenmeyer flasks. Seed cultures were maintained in $\mathrm{YM}$ liquid medium on a rotary shaker at $95 \mathrm{rpm}$ for a minimum of 5 days (to a maximum of 7 days) at $28 \pm 2{ }^{\circ} \mathrm{C}$ for biomass enrichment $\left(\geq 5 \times 10^{8}\right.$ cells $\left.\mathrm{mL}^{-1}\right)$ prior to inoculations. 
The axenic state of seed cultures was confirmed by light microscopy (at $400 \times$ magnification on an Olympus CX21LED, Philippines) at every stage of the cultivation process.

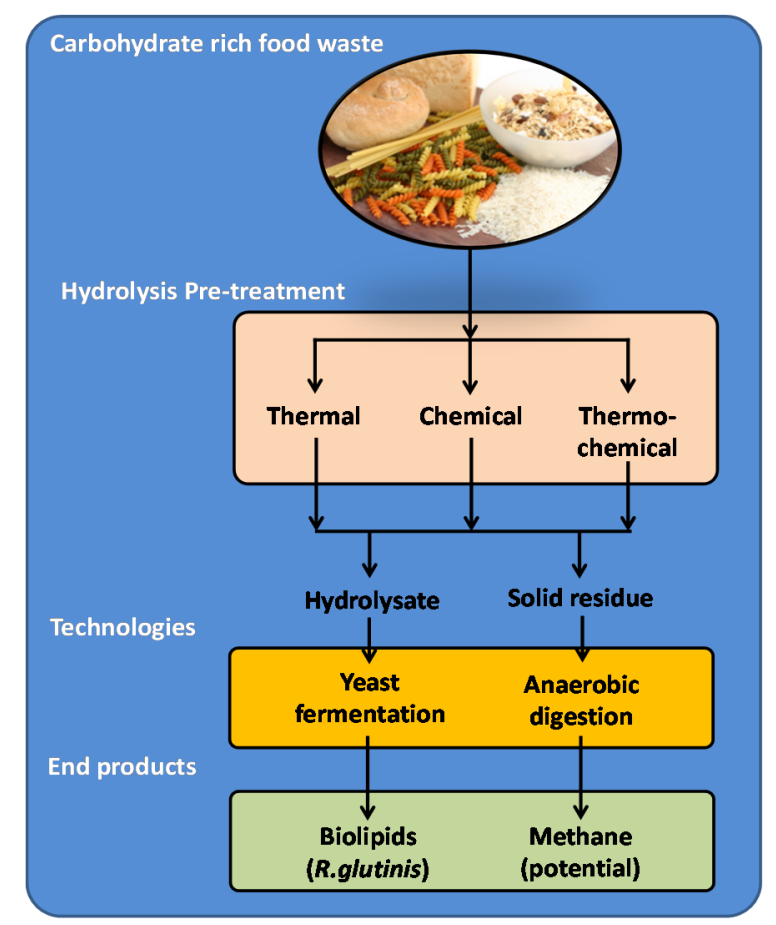

Figure 1. Proposed integrated bio-refining approach for carbohydrate-rich food waste recycling for biofuels

\subsection{Bio-Refining of Carbohydrate-Rich Food Waste}

CRFW was a mixture of bread $(25.9 \%)$, oats $(29.8 \%)$, cooked pasta $(27.7 \%)$ and boiled rice $(16.6 \%)$ which was homogenised $\left(<1 \mathrm{~cm}^{3}\right.$ particle size) by grinding (PB7600s MultiBlender ${ }^{\mathrm{TM}}$ Pro-Sunbeam). The homogenized CFRW was characterized for various parameters before and after pre-treatment as detailed in Section 2.3.

\subsubsection{Hydrolysis of CRFW}

Feed slurry was prepared by adding $250 \mathrm{~g}$ CRFW to $1 \mathrm{~L}^{-1}$ of deionised (DI) water and refrigerated at $4{ }^{\circ} \mathrm{C}$ for $24 \mathrm{~h}$ to allow passive leaching of nutrients, whilst minimising microbial growth. The resultant mixtures represented the physical control (PCon) throughout the study (i.e., $4{ }^{\circ} \mathrm{C}$ 24 h-leachate). The slurry was hydrolyzed using three different approaches:

(i) Chemical hydrolysis (Ch) — acidic hydrolysis of the CRFW slurry at a pH of $3 \pm 0.01$ adjusted with $2 \mathrm{M} \mathrm{HCl}$ (Sigma Aldrich, Australia) for $24 \mathrm{~h}$ at room temperature $\left(25^{\circ} \mathrm{C}\right)$;

(ii) Thermal hydrolysis (Th) — autoclaving of the CRFW slurry using a standard moisture-heat procedure of $121{ }^{\circ} \mathrm{C}$ at $1013.25 \mathrm{hPa}$ for $30 \mathrm{~min}$ (Tomy, VWR International, Murarrie, QLD 4172, Australia);

(iii) Thermochemical hydrolysis (TCh) — a combined double hydrolysing procedure, where the chemical hydrolysis of the slurry preceded the thermal hydrolysis. 
Following hydrolysis, hydrolysates were centrifuged at $15,900 \times \mathrm{g}$ for $20 \mathrm{~min}$ with $10 \mathrm{~min}$ deceleration at $4{ }^{\circ} \mathrm{C}$ (Avanti $囚 \mathrm{~J}-26 \mathrm{XPI}$, Beckman Coulter, USA). The supernatants (hydrolysates) were decanted into sterilised $2 \mathrm{~L}$ Simax bottles for $R$. glutinis enrichment (as detailed in Section 2.2.2). Prior to inoculation with $R$. glutinis, total carbohydrate was determined for each hydrolysate (as detailed in Section 2.3) and $\mathrm{pH}$ was adjusted to 4 with addition of either $1 \mathrm{M} \mathrm{HCl}$ or $\mathrm{NaOH}$ (WP-81, Thermofisher Scientific, Australia). pH probes were cleaned and sterilized with an ethanol wash before each measurement. Residual solids (RS) were characterized for biogas and corresponding bioenergy (methane) potential was estimated using Buswell equation (as detailed in Section 2.3.3).

\subsubsection{Cultivation of $R$. Glutinis in Hydrolysates for Bio-Product Development}

$500 \mathrm{~mL}$ of undiluted hydrolysates of each treatment were inoculated with $R$. glutinis $\sim 1.0 \times 10^{9}$ cells in sterile $1 \mathrm{~L}$ Simax reagent bottles with a modified polypropylene cap for aeration and ventilation. The culture bottles were maintained with a continuous airflow of $130 \pm 0.7 \mathrm{~L} \mathrm{~h}^{-1}$ at $28 \pm 2{ }^{\circ} \mathrm{C}$ for 7 days. Filtered air $0.45 \mu \mathrm{m}$ syringe filter (Advantec, VWR International) was supplied via a $2 \mathrm{~mL}$ glass pipette connected to a Precision Air Pump 7500 (Aqua One, Local Aquarium, Townsville, Australia) and venting occurred via pipette tips filled with cotton wool. Although photo periods and light intensities are not limiting factors for lipid accumulation in saprophytic microorganisms, R. glutinis experiments were conducted under a 12:12 light:dark cycle as cultivation was carried out in the algal culture room at the North Queensland Algal Culture Identification Facilities (NQAIF, James Cook University, Australia). Growth of $R$. glutinis was monitored daily by cell count (Neubauer improved bright-line haemocytometer) and measuring dry weight (DW) gravimetrically [25] at days 0 , 1, 3, 5 and 7. Total carbohydrates were measured using the UV-sulphuric acid method [26] at days 1, 4 and 7 and the system pH (portable pH meter-Oaklon ${ }^{\circledR}$, Singapore) was measuered at days 0 and 7. Experiments were performed in triplicates and all sampling occurred in a sterile laminar flow cabinet (AES Environmental Pty LTD fitted with HEPA filter, Australia) to minimise contamination. Rhodotorula glutinis biomass was harvested from hydrolysates by centrifugation, was freeze-dried and extracted for transesterification into fatty acid methyl esters (FAME) [27]. In brief, $30 \pm 4 \mathrm{mg}$ lyophilised $R$. glutinis biomass was measured into $8 \mathrm{~mL}$ Teflon capped glass vials (Supelco, Sigma-Aldrich). An equal volume $(50 \mathrm{mg}$ ) of $0.5 \mathrm{~mm}$ zirconium oxide beads (Next Advance) was added to the biomass, serving as abrasive particles for mechanical cell wall disruption. $2 \mathrm{~mL}$ of freshly prepared methylation mixture, HPLC-grade methanol and acetyl chloride $(95: 5 \mathrm{v} / \mathrm{v})$, was added and supplemented with $300 \mu \mathrm{L}$ internal standard solution (Nonadecanoic acid-C19:0, $0.2 \mathrm{mg} \mathrm{mL}{ }^{-1}$ HPLC-methanol). The methylated-biomass mixture was vortexed at 2,200 rpm for $30 \mathrm{~s}$ at $30 \mathrm{~s}$ intervals (Schneiter and Daum, 2006). Once homogenised, vials were placed into a block heater (Ratek DBH30, Australia) at $100{ }^{\circ} \mathrm{C}$ for $60 \mathrm{~min}$ to facilitate transesterification of fatty acids to methyl esters. Heated samples were allowed to cool to room temperature, before adding $1 \mathrm{~mL}$ non-polar organic solvent $(0.01 \% \mathrm{BHT} \mathrm{w} / \mathrm{W}$ HPLC-Hexane) and mixing by inversion. Sample vials were replaced into the warm block heater for $60 \mathrm{~s}$, enabling the formation of a miscible mixture. Once cooled, addition of $1 \mathrm{~mL}$ UltraPure water (MilliQ, Life Technologies) separated the two phases. The upper FAME-hexane mixture was collected and filtered through a $0.2 \mu \mathrm{m}$ PTFE filter (Agilent) prior to its injection into GC vials. Gas chromatography determination of FAME profiles were carried out on an Agilent 7890 GC with flame 
ionisation detector (FID) and Electron Ionisation (EI) Turbo Mass Spectrometer (MS) (Agilent 5975C, Agilent Technologies Australia Pty Ltd). A DB-23 column with cyanopropyl stationary phase $(60 \mathrm{~m} \times 0.55 \mathrm{~mm} \mathrm{id} \times 0.15 \mu \mathrm{m})$ with $\mathrm{He}_{2}(\mathrm{~g})$ injection $\left(33 \mathrm{~cm} \mathrm{~s}^{-1}\right.$ at $\left.50{ }^{\circ} \mathrm{C}\right)$ at $230 \mathrm{kPa}$ was used for sample separation. Constant inlet temperatures for injector and FID were maintained at $150{ }^{\circ} \mathrm{C}$ and $250{ }^{\circ} \mathrm{C}$ with split injection of $1 / 50$, respectively. Oven and column temperature settings were based on instrumental protocols by the manufacturer. Unknown FAME profiles were determined via comparison of peaks and retention times of pure external standards (C8-C24, Sigma-Aldrich), whilst the recovery potential was corrected using a factor derived from the known concentrations of nonadecanoic acid (C19:0) used as internal standards. Review of the literature and supporting data from procedure blanks confirmed that C19:0 is not produced by $R$. glutinis $[23,28,29]$.

\subsection{Analytical Procedure}

\subsubsection{Reagents and Standard Calibration Gases}

All chemicals and reagents were obtained from Sigma-Aldrich, Australia. The calibration $\mathrm{CH}_{4}$ gases (i.e., 10\%-50\%), helium and compressed air $\left(\mathrm{N}_{2}-78.08 \%\right.$ and $\mathrm{O}_{2}-20.94 \%$ ) for GC-TCD-FID were supplied by BOC a member of the Linde group, Townsville, Australia. All gases were ISO certified for purity.

\subsubsection{Characterization of CRFW}

$25 \pm 2$ g CRFW and RS were freeze-dried over $48 \mathrm{~h}$ (Virtis benchtop 2K, VWR International, Australia). The subsequent lyophilised products were then homogenised in pre-dried $\left(105 \pm 2{ }^{\circ} \mathrm{C}\right.$ for $\left.4 \mathrm{~h}\right)$ porcelain mortars into a fine powder, which were passed through a $1 \mathrm{~mm}^{2}$ stainless steel mesh to exclude large fragments for CHNS-O analysis. The sample analysis was outsourced to Organic Elemental Analysis Laboratories (OEA Laboratory Ltd., Cornwall, UK). Total and volatile solids (TS and VS, [25]) for CRFW and RS were measured and moisture contents were back calculated (moisture $\%=100-\mathrm{TS}$ ).

\subsubsection{Calculating Bio-diesel and Bio-energy Potential of Bio-refined CRFW}

The potential physicochemical properties of biodiesel were calculated based on levels of (un)saturation and carbon length of the individual FAMEs using established models [30,31]. For this study, the values of cetane $(\mathrm{CN})$, kinematic viscosity (v), density ( $\rho$ ), and higher heating values $\left(\mathrm{HHV}_{\mathrm{B}}\right)$ of biodiesel were calculated using Equations 1-4, respectively.

$$
\begin{gathered}
C N=\sum_{i}\left(-7.8+0.302 \times M_{i}-20 \times N\right) \\
\ln \left(v_{i}\right)=-12.503+2.496 \times \ln \left(M_{i}\right)-0.178 \times N \\
\rho_{i}=0.8463+\frac{4.9}{M_{i}}+0.0118 \times N \\
H H V_{B(i)}=46.19+\frac{1794}{M_{i}}-0.21 \times N
\end{gathered}
$$


where $\mathrm{N}_{\mathrm{i}}, \mathrm{M}_{\mathrm{i}}$, and $\mathrm{D}_{\mathrm{i}}$ represent the percentage, molecular weight and number of double bonds in the respective $i^{\text {th }}$ FAME.

Based on the elemental analysis, theoretical biogas ( $\left.B_{\text {th }}\right)$ yields of RS were calculated using Equations 5 and 6 [32] and compared with actual $\mathrm{CH}_{4}$ potential as reported in literature [33]:

$$
\begin{gathered}
B_{t h}\left[\frac{m^{3}}{k g_{V S}}\right]=\frac{a * 22.415}{12 a+b+16 c+14 d} \\
M_{t h}\left[\frac{m^{3}}{k g_{V S}}\right]=\frac{\left(\frac{4 a+b-2 c-3 d}{8}\right) * 22.415}{12 a+b+16 c+14 d}
\end{gathered}
$$

where, a: carbon-C\%; b: hydrogen-H\%; c: oxygen-O\% d: nitrogen-N\%.

\section{Results and Discussion}

\subsection{Characteristics of Hydrolysates from Pre-treated CRFW}

Hydrolysis pre-treatment of CRFW converts complex organic structures into simpler molecules (mainly sugars) making them readily available for microbial conversion [34]. Hydrolysed CRFW had a $\mathrm{pH}$ range of 3.77-5.79 based on the different pre-treatments. Therefore, $\mathrm{HCl}$ or $\mathrm{NaOH}$ were used to reduce/increase the $\mathrm{pH}$ of hydrolysates for cultivation of $R$. glutinis. Buffering capacities of the hydrolysates differed, requiring variable volumes and concentrations of acids/bases. Compared to Pcon leachate total carbohydrate content $\left(\sim 45 \mathrm{mg}\right.$ eq-Gluc. $\left.{ }^{-1}\right)$, carbohydrate release was higher $(\sim 25 \%$; i.e., $\sim 65 \mathrm{mg}$ eq-Gluc. $\mathrm{g}^{-1}$ ) in the thermal hydrolysis leading to a change in leachate $\mathrm{pH}$ from 4.01 to 5.79, whereas, the $\mathrm{Ch}$ or TCh pre-treatments did not improve total carbohydrate release from CRFW. These results are in contrast to acid-hydrolysed fruit and vegetable waste which released more carbohydrates than when subjected to alkali or Th hydrolysis [35]. However, another study showed, that, whilst acid directly hydrolysed/solubilised starch and hemicellulose from CRFW, thermal hydrolysis was more efficient [36]. Furthermore, Th hydrolysis also simultaneously reduces pathogen levels and viscosity of the medium [37], which resulted in easier handling of RS and R. glutinis cultivation.

\subsection{Growth of R. glutinis in CRFW Hydrolysates}

Whilst final cell concentrations were similar for Th-, Ch- and Pcon-treated CRFW, growth responses of $R$. glutinis in the early stages of cultivation varied. Foam formation occurred in the culture based on TCh-hydrolysate causing $>80 \%$ loss of of $R$. glutinis cells and leading to termination on day 4 (Figure 2). Foaming could be due to high aeration and inhibited fermentation processes led to protein degradation in the systems. Culture growth was accompanied by a shift in system $\mathrm{pH}$ from 4 to $7.62 \pm 0.17$ and $6.36 \pm 0.02$ in Pcon and Th cultures, respectively. Growth of $R$. glutinis was comparable between Th and Ch hydrolysates, peaking within 2 days. However, Ch hydrolysates better supported the growth of $R$. glutinis and achieved maximum cell counts within $24 \mathrm{~h}$. On the other hand, Th hydrolysates contained more carbohydrates, which were expected to provide higher $R$. glutinis biomass.

All systems reached $R$. glutinis densities of 2.60-2.67 $\times 10^{8}$ cells within 4 days are remained stable during stationary phase until day 7 , which is consistent with another report [15]. In terms of 
biomass yield, $3.42-3.61 \mathrm{~g}$ DW biomass $\mathrm{L}^{-1}$ was measured from Pcon $\left(3.61 \pm 0.25 \mathrm{~g} \mathrm{DW}_{\text {biomass }} \mathrm{L}^{-1}\right)$, Ch $\left(3.42 \pm 0.41 \mathrm{~g} \mathrm{DW}_{\text {biomass }} \mathrm{L}^{-1}\right)$ and Th $\left(3.53 \pm 0.94 \mathrm{~g} \mathrm{DW}\right.$ biomass $\left.\mathrm{L}^{-1}\right)$ hydrolysates and no significant differences were observed between the cultivation media. Our results were also comparable to biomass yields of 4.3-6.9 gDW biomass. $\mathrm{L}^{-1}$ achieved when cultivating $R$. glutinis on carbon sources such as glucose, xylose, arabinose at a $\mathrm{pH} 5.8$ and temperature $28^{\circ} \mathrm{C}$ [38]. Carbohydrate analysis at harvest indicated that secondary or continuous cultivation of $R$. glutinis over a longer period may be possible, as $\sim 29 \%$ (from Pcon and TCh hydrolysates) and $\sim 24 \%$ (from Ch hydrolysate) of carbohydrates were assimilated within 7 days. As nitrogen was likely limiting biomass yield and also carbon utilization [23], nitrogen supplementation could enhance these process performance criteria.

In the context of biofuel potential, for which green microalgae have been identified as a potential biomass source [30] total lipid contents of $14.05 \%$ (Chlorella sp.) of dryweight biomass have been reported [39]. In contrast, achieved CRFW hydrolysate-cultivated $R$. glutinis biomass yields were $3.5 \times$ higher $\left(\sim 3.5 \mathrm{~g} \mathrm{DW} \mathrm{L}{ }^{-1}\right.$ with a total lipid content of $\left.40 \%\right)$ compared to typical yields of green microalgae cultivated in open system suspension cultures [40].

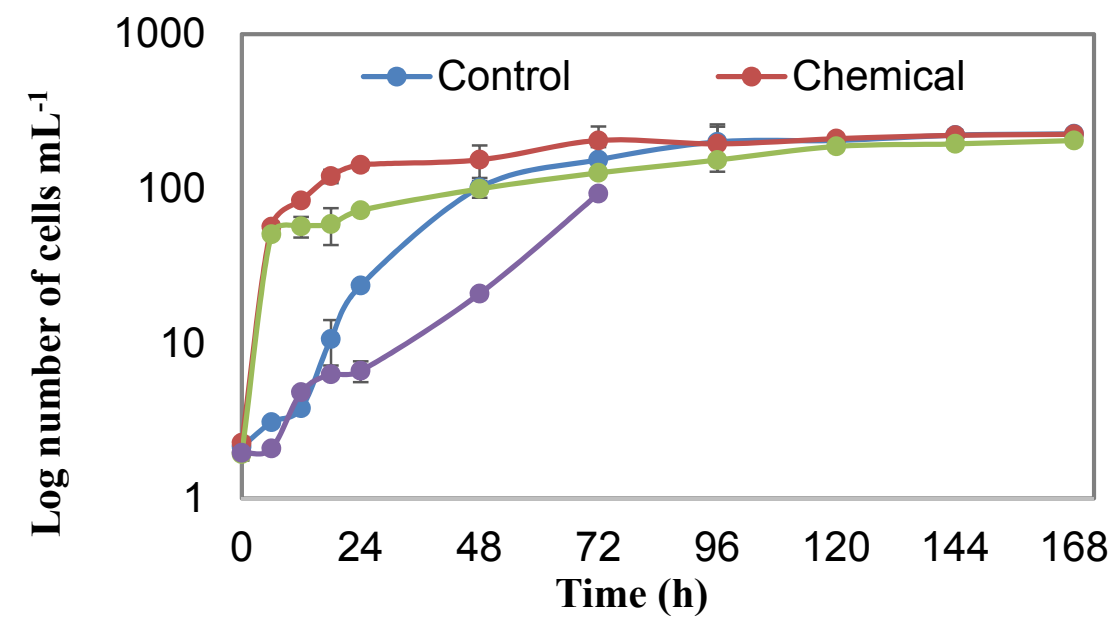

Figure 2. Growth of $R$. glutinis in carbohydrate-rich food waste (CRFW) hydrolysates.

\subsubsection{FAME Profile of $R$. glutinis Cultivated in CRFW Hydrolysates}

The total fatty acid (TotFA) contents and FAME profiles of $R$. glutinis enriched in CRFW hydrolysates are shown in Table 1. TotFA contents were $41.79 \pm 12.6,38.05 \pm 8.2$ and $65.56 \pm 30.9$ $\mathrm{mg}_{\text {TotFA. }} \mathrm{g}^{-1} \mathrm{DW}$ biomass for Pcon-, Ch- and Th-cultivated $R$. glutinis, respectively. TotFA content achieved for Th-cultivated $R$. glutinis is comparable to reported values for its biomass derived from food waste and wastewater (62-63 mg TotFA.g ${ }^{-1} \mathrm{DW}_{\text {biomass }}$ [20]). A positive correlation between medium carbohydrate content and TotFA content of the derived biomass was also demonstrated in Braunwald, Schwemmlein [23]. Compared to Pcon, biomass TotFA of Ch-cultivated R. glutinis was lower, suggesting that stored fatty acids may be re-utilized (i.e., lipid turnover) during prolonged cultivation [41]. This is consistent with the literature, reporting a $63 \%$ reduction of TotFA content (i.e., from 190 (3rd day) to 120 (5th day) gg Lipids. $^{-1} \mathrm{DW}_{\text {biomass }}$ ) for $R$. glutinus with prolonged cultivation when cultivated in wastewater [42].

The FAME profiles of CRFW hydrolysate-cultivated $R$. glutinis are shown in Figure 3. Mono- and poly-unsaturated fatty acids (MUFA and PUFA, respectively) accounted for $61 \%-67 \%$, while 
saturated fatty acids (SFA) were only $32 \%-39 \%$ of the biomass. While general fatty acid profiles were similar, the percentage distributions of fatty acids differed. Highest MUFA and PUFA contents were achieved in R. glutinis cultivated in Th and PCon hydrolysates, respectively. Irrespective of cultivation medium, fatty acid profiles and elongation and desaturation patterns were typical for $R$. glutinis and de novo lipid synthesis [43]. In line with other reports, oleic acid ( $\left.\mathrm{C}_{18: 1}\right)$ and linoleic acid ( $\left.\mathrm{C}_{18: 2}\right)$ were the main MUFA and PUFA of $R$. glutinis cultivated in CRFW hydrolysates [16,23,44]. Oleic acid (C18:1) accounted for $\sim 42 \%$ in Th-cultivated $R$. glutinis biomass, whilst the levels were 35 and 44\% lower in Ch- and Pcon cultivates, respectively. On the other hand, palmitic acid (C16:0) was elevated by $43 \%$ in Ch-cultures, while contents were similar for Th- and Pcon cultivated $R$. glutinis biomass. Highest levels of C18:2 (26\%) were achieved in Pcon-cultivated R. glutinis declining by 37.5 and $65 \%$ with hydrolysis treatment (Ch- and Th-cultures, respectively) with an opposite trend observed for stearic acid (C18:0). A-linolenic acid (C18:3) was highest in Ch-cultures, slightly lower in Pcon and lowest in Th-cultivated biomass, with an opposite trend observed for palmitoleic acid (C16:1). While margaric acid (C17:0) was not detectable in Th-cultvated $R$. glutinis, heptadecenoic acid (C17:1), C16:1 and myristic acid (C14:0) was observed in small amounts under all cultivation conditions. Overall, the FAME profiles obtained from all $R$. glutinis cultures are considered to be ideal precursors for the biofuel industry [44-46].

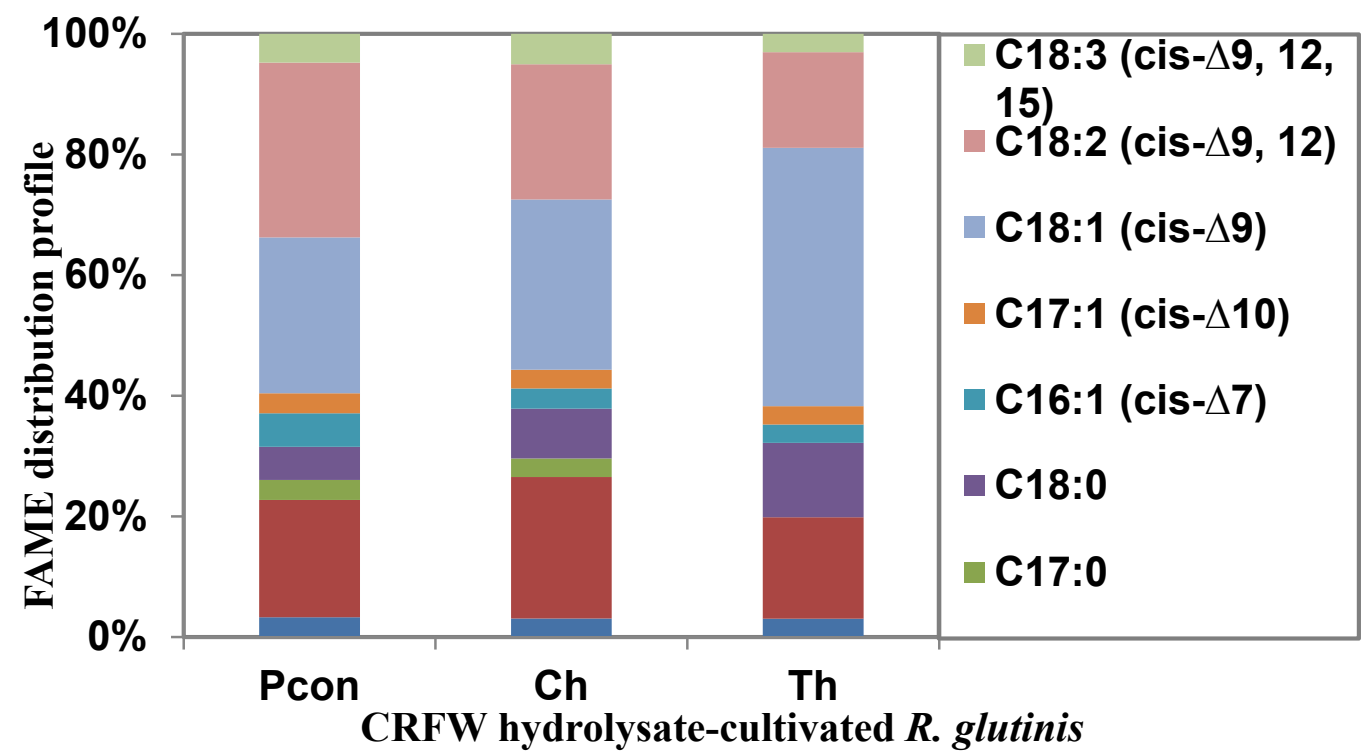

Figure 3. Fatty acid methyl ester (FAME) profiles (\%) of $R$. glutinis grown in CRFW hydrolysates.

Table 1. Total fatty acid contents and distribution of fatty acid categories in R. glutinis cultivated in CRFW hydrolysates.

\begin{tabular}{lccc}
\hline \multirow{2}{*}{\multicolumn{1}{c}{ Particulars }} & \multicolumn{3}{c}{ Pre-treatment of CRFW } \\
\cline { 2 - 4 } & PCon & Ch & Th \\
\hline Fatty Acids (mg g ${ }^{-1}$ DW) & $41.79 \pm 12.67$ & $38.05 \pm 8.23$ & $65.56 \pm 30.91$ \\
Branching Fatty Acid (\%) & $9.88 \pm 6.02$ & $3.79 \pm 2.18$ & $<3$ \\
Saturated Fatty Acid (\%) & $37.81 \pm 7.41$ & $39.5 \pm 2.78$ & $32.53 \pm 11.50$ \\
Monounsaturated Fatty Acid (\%) & $28.64 \pm 12.90$ & $31.82 \pm 12.77$ & $51.65 \pm 34.85$ \\
Polyunsaturated Fatty Acid (\%) & $33.55 \pm 20.81$ & $28.67 \pm 12.82$ & $15.82 \pm 0.98$ \\
\hline
\end{tabular}




\subsubsection{Biodiesel Potential of $R$. glutinis Enriched from CRFW Hydrolysates}

Biodiesel properties calculated based on FAME profiles of $R$. glutinis biomass cultivated using CRFW-hydrolysates yielded a density of $\rho \sim 0.79$ to $0.85 \mathrm{~g} \mathrm{~cm}^{-3}$ and mean HHVs of 35.79-39.36 MJ.kgThe HHVs were 14\%-25\% lower than the fossil fuel-derived diesel [47]. Calculated CNs were comparable with the biodiesel standard (ASTM D6751-02), i.e., $\mathrm{CN}>51$, but higher than demanded standards for fossil fuel-derived diesel (ASTM D975; CN 40-50). In addition, the calculated viscosity $(v)$ for the biomass was within the standard limits $\left(v-1.3-4.1 \mathrm{~mm}^{2} \mathrm{~S}^{-1}\right)$ for fossil fuel-derived diesel (Table 2). In general, higher CN, $\rho$ and HHV are correlated with MUFA content of TotFA of R. glutinis harvested from Th hydrolysates. If required, these properties can be tailored to meet the required standards in downstream processes or the biofuel can be used as a diesel blend [48]. While higher SFA contents correlate with higher $\mathrm{CN}$, contributing to shorter ignition delay times and improved oxidative stability of final products [41], cold use properties decline, as the biofuels become more viscous with decreasing temperatures [30]. It should be recognized that at present only B5 (5\% biodiesel content) fuels are being used, but the calculated fatty acid-derived properties of CRFW hydrolysate cultivated $R$. glutinis indicates that higher mixing ratios are possible without adverse effects on key fuel properties [30]. In the context of biodiesel production, the fatty acid profiles and contents of the red yeast $R$. glutinis cultivated on CRFW hydrolysates are comparable to those in green algae [40], but biomass yields $(3.5 \times$ higher $)$ and total lipid contents $(3-4.5 \times$ higher $)$ were superior than green algal biomass cultivated under phototrophic conditions in open systems. Together with the potential secondary or continuous cultivation option for R. glutinis cultivation in CRFW hydrolysates, this would translate into a potentially much smaller production area foot print when compared to green algal biodiesel. However, required energy inputs for the cultivation of the respective biomass will need detailed investigation.

Table 2. Standard biodiesel properties as set by ASTM D6751-02 and EN 14214 and FAME-based calculated biodiesel properties of $R$. glutinis cultivated on CRFW hydrolysate.

\begin{tabular}{lcccc}
\hline & $\mathbf{C N}$ & $\boldsymbol{v}$ & $\boldsymbol{\rho}$ & HHV \\
\hline ASTM D6751-02 & $\geq 47$ & $1.9-6.0$ & $0.86^{*}$ & NA \\
EN 14214 & $\geq 51$ & $3.5-5.0$ & $0.86-0.90$ & $35^{* *}$ \\
PCon & $51.06 \pm 0.12$ & $3.59 \pm 0.05$ & $0.79 \pm 0.03$ & $35.76 \pm 1.23$ \\
Ch & $56.92 \pm 0.36$ & $3.99 \pm 0.9$ & $0.84 \pm 0.02$ & $38.19 \pm 1.11$ \\
Th & $62.35 \pm 0.45$ & $4.46 \pm 0.12$ & $0.86 \pm 0.06$ & $39.36 \pm 1.36$ \\
\hline
\end{tabular}

Note: * typical values; ** set by the DIN 51900.

\subsection{Characteristics of Residual Solids and Bioenergy Potential}

The elemental composition of RS from hydrolysis pre-treatments are given in Table 3. Hydrolysis pre-treatment had no large effect on C, O, N, H, and S contents. Also, high VS ( $96 \%-97 \%)$ and TS contents suggest that the RS is suitable for bioconversion. The calculated $\mathrm{C} / \mathrm{N}$ ratios of the RS were between 15-19, similar to those in the organic fraction of municipal solid waste; OF-MSW [49]. For anaerobic digestion, optimal $\mathrm{C} / \mathrm{N}$ ratios of 27-32 were recommended in order to avoid any build-up of ammonia and associated toxicity effects in reactors that subsequently affect $\mathrm{CH}_{4}$ yield [49]. 
Theoretical $\mathrm{CH}_{4}$ yields could be lower, i.e., $0.16-0.18 \mathrm{~m}^{3} \mathrm{CH}_{4} . \mathrm{kgVS}^{-1}$, compared to reported literature values of $0.18-0.24 \mathrm{~m}^{3} \mathrm{CH}_{4} \cdot \mathrm{kgVS}^{-1}$ reported for FW/OF-MSW [32]. This lower $\mathrm{CH}_{4}$ potential could be due to the removed carbohydrate contents of the waste, supplied as hydrolysates for R. glutinis cultivation in the previous experiments, which has been reported in association with the removal of organic material removal following pre-treatment of organic waste [50]. In the context of the proposed bio-refinery concept, the actual $\mathrm{CH}_{4}$ potential of recycled $\mathrm{RS}$ from the hydrolysates of CRFW will require experimental validation.

Table 3. Characteristics of residual solids of CRFW hydrolysates.

\begin{tabular}{lcccc}
\hline Parameters & Pcon & Ch & Th & TCh \\
\hline Carbon & $41.86 \pm 0.81$ & $43.02 \pm 0.32$ & $42.27 \pm 0.28$ & $44.13 \pm 0.31$ \\
Nitrogen & $2.68 \pm 0.22$ & $2.83 \pm 0.18$ & $2.29 \pm 0.26$ & $3.06 \pm 0.31$ \\
Hydrogen & $6.41 \pm 0.23$ & $6.4 \pm 0.37$ & $6.46 \pm 0.18$ & $6.63 \pm 0.09$ \\
Sulphur & $0.12 \pm 0.02$ & $0.14 \pm 0.01$ & $<0.1$ & $0.19 \pm 0.01$ \\
Oxygen & $48.95 \pm 1.11$ & $47.62 \pm 0.59$ & $48.99 \pm 0.83$ & $46 \pm 1.21$ \\
C/N ratio & $15.62 \pm 0.58$ & $15.23 \pm 0.67$ & $18.46 \pm 0.38$ & $14.42 \pm 0.79$ \\
Total solids & $74.13 \pm 2.71$ & $75.92 \pm 1.63$ & $74.68 \pm 1.42$ & $75.15 \pm 2.35$ \\
Volatile solids & $96.76 \pm 0.97$ & $95.75 \pm 1.25$ & $95.97 \pm 1.54$ & $97.1 \pm 1.80$ \\
\hline
\end{tabular}

Note: All values are in $\%$.

\section{Conclusions}

The proposed integrated yeast fermentation and anaerobic digestion process appears to be a promising approach for the bio-refining of CRFW for biolipids and bioenergy production. Biomass yields, total fatty acid content and profile, as well as calculated important diesel characteristics, render R. glutinis a suitable alternative to green microalgal biodiesel when cultivated on CRFW hydrolysates under controlled conditions, potentially requiring a fraction of the cultivation foot print. Ch hydrolysates provided better biomass yields, however biodiesel properties and FAME yields were higher with the Th hyrolysate cultivates. In addition, solid residue from the Th pre-treatment was estimated with higher methane potential. Therefore, Th hydrolysis of CRFW followed by R. glutinis cultivation under buffered condition should be recommended for further investigation.

Further research is required with regards to outdoor cultivation suitability and competitiveness for CRFW recycling. Residual solids characteristics and theoretical yields show promise for additional energy benefits that can be derived through the bioconversion of CRFW. However, the full potential of this novel integrative bio-refinery concept for CRFW hydrolysates and residual solids requires further opitmization of cultivation conditions and field experimentation to validate biomass yields, total lipids yields and actual methane production, as well as a full characterization of the resulting biodiesel characteristics and quantities, which will lay the foundation for a comprehensive techno-economic analysis and energy requirements of bioenergy generation using different feedstocks. In addition, unutilized sugars from $R$. glutinis fermentation could be potentially re-cycled within the fermentation system or anaerobically digested for making this technology more energy efficient and economically viable. Furthermore, heat and power generated from biogas combustion could be potentially re-routed for hydrolysis pre-treatments which would benefit the proposed integrated bio-refining approach. 


\section{Acknowledgments}

The project was supported by the Advanced Manufacturing Cooperative Research Centre (AMCRC), funded through the Australian Government's Cooperative Research Centre Scheme, grant number 2.3.4. The funders had no role in study design, data collection and analysis or preparation of the manuscript and have provided permission to publish. This research is part of the MBD Energy Research and Development program for Biological Carbon Capture and Storage.

\section{Author Contributions}

All authors contributed to generating this manuscript. The research data were generated within the Honours research project by Hoang-Tuong Nguyen Hao under the supervision of Obulisamy Parthiba Karthikeyan and Kirsten Heimann.

\section{Conflicts of Interest}

The authors declare no conflict of interest.

\section{References}

1. Cherubinia, F.; Ulgiatib, S. Crop residues as raw materials for biorefinery systems-A LCA case study. Appl. Energy 2010, 87, 47-57.

2. Demirbas, M.F. Biorefineries for biofuel upgrading: A critical review. Appl. Energy 2009, 86, S151-S161.

3. Sadhukhan, J.; Ng, K.S.; Hernandez, E.M. Biorefineries and Chemical Processes: Design, Integration and Sustainability Analysis; Wiley: Chichester, West Sussex, UK, 2014.

4. Wright, M.M.; Brown, R.C. Comparative economics of biorefi neries based on the biochemical and thermochemical platforms. Biofuel. Bioprod. Bior. 2007, 1, 49-56.

5. Menon, V.; Rao, M. Trends in bioconversion of lignocellulose: Biofuels, platform chemicals \& biorefinery concept. Prog. Energy Combust. Sci. 2012, 38, 522-550.

6. Kamm, B.; Gruber, P.R.; Kamm, M. Biorefineries-Industrial Processes and Products: Status quo and Future Directions; Wiley-VCH: Weinheim, Germany, 2006.

7. Parfitt, J.; Barthel, M.; Macnaughton, S. Food waste within food supply chains: Quantification and potential for change to 2050. Philos. T. R. Soc. B 2010, 365, 3065-3081.

8. Wang, L.J. Production of bioenergy and bioproducts from food processing wastes: A review. T. Am. Soc. Agr. Biol. Eng. 2013, 56, 217-229.

9. Smith, K.; O'Farrel, K.; Brindley, F. Waste and recycling in Australia 2011; Department of Sustainability, Environment, Water, Population and Communities: Sydney, Australia, 2012.

10. Grosso, M.; Motta, A.; Rigamonti, L. Efficiency of energy recovery from waste incineration, in the light of the new Waste Framework Directive. Waste Manage. 2010, 30, 1238-1243.

11. Liang, S.; McDonald, A.G.; Coats, E.R. Lactic acid production with undefined mixed culture fermentation of potato peel waste. Waste Manage. 2014, 34, 2022-2027.

12. Sun, Z.; Li, M.; Qi, Q.; Gao, C.; Lin, C.S. Mixed food waste as renewable feedstock in succinic acid fermentation. Appl. Biochem. Biotechnol. 2014, 174, 1822-1833. 
13. Koutinas, A.A.; Vlysidis, A.; Pleissner, D.; Kopsahelis, N.; Lopez Garcia, I.; Kookos, I.K.; Papanikolaou, S.; Kwan, T.H.; Lin, C.S. Valorization of industrial waste and by-product streams via fermentation for the production of chemicals and biopolymers. Chem. Soc. Rev. 2014, 43, 2587-2627.

14. Cheirsilp, B.; Suwammarat, W.; Niyomdecha, R. Mixed culture of oleaginous yeast Rhodotorula glutinis and microalga Chlorella vulgaris for lipid production from industrial wastes and its use as biodiesel feedstock. New Biotechnol. 2011, 28, 362-368.

15. Saengea, C.; Cheirsilpb, B.; Suksarogea, T.T.; Bourtoomc, T. Potential use of oleaginous red yeast Rhodotorula glutinis for the bioconversion of crude glycerol from biodiesel plant to lipids and carotenoids. Proc. Biochem. 2011, 46, 210-218.

16. Schneider, T.; Graeff-Hönninger, S.; French, W.T.; Hernandez, R.; Claupein, W.; Holmes, W.E.; Merkt, N. Screening of Industrial Wastewaters as Feedstock for the Microbial Production of Oils for Biodiesel Production and High-Quality Pigments. J. Combust. 2012, 153410:1-153410:9.

17. Hu, C.; Zhao, X.; Zhao, J.; Wu, S.; Zhao, Z.K. Effects of biomass hydrolysis by-products on oleaginous yeast Rhodosporidium toruloides. Bioresource Technol. 2009, 4843-4847.

18. Ratledge, C. Yeasts, moulds, algae and bacteria as sources of lipids. In Technological Advances in Improved and Alternative Sources of Lipids; Kamel, B.S., Kakuda, Y., Eds.; Blackie Academic and Professional: London, UK, 1994.

19. Beopoulos, A.; Desfougeres, T.; Sabirova, J.; Zinjarde, S.; Neuveglise, C.; Nicaud, J.M. The hydrocarbon-degrading oleaginous yeast Yarrowia lipolytica. In Handbook of Hydrocarbon and Lipid Microbiology; Timmis, K.N., Ed.; Springer: New York, NY, USA, 2009.

20. Chi, Z.; Zheng, Y.; Jiang, A.; Chen, S. Lipid production by culturing oleaginous yeast and algae with food waste and municipal wastewater in an integrated process. Appl. Biochem. Biotechnol. 2011, 165, 442-453.

21. Xue, F.; Gao, B.; Zhu, Y.; Zhangm, X.; Feng, W.; Tan, T. Pilot-scale production of microbial lipid using starch wastewater as raw material. Bioresource Technol. 2010, 101, 6092-6095.

22. Beopoulos, A.; Nicaud, J.M.; Gaillardin, C. An overview of lipid metabolism in yeasts and its impact on biotechnological processes. Appl. Microbiol. Biotechnol. 2011, 90, 1193-1206.

23. Braunwald, T.; Schwemmlein, L.; Graeff-Hönninger, S.; French, W.T.; Hernandez, R.; Holmes, W.E.; Claupain, W. Effect of different $\mathrm{C} / \mathrm{N}$ ratios on carotenoid and lipid production by Rhodotorula glutinis. Appl. Microbiol. Biotechnol. 2013, 97, 6581-6588.

24. Saenge, C.; Cheirsilp, B.; Suksaroge, T.T.; Bourtoom, T. Effificent concomitant production of lipids and carotenoids by oleaginous red yeast Rhodotorula glutinis cultured in palm oil mill effluent and application of lipids for biodiesel production. Biotechnol. Bioproc. Eng. 2011, 16, 23-33.

25. APHA. Standard Methods for the Examination of Water and Wastewater, 21st ed.; American Public Health Organization: Washington DC, USA, 2005.

26. Albalasmeh, A.A.; Berhe, A.A.; Ghezzehei, T.A. A new method for rapid determination of carbohydrate and total carbon concentrations using UV spectrophotometry. Carbohydr. Polym. 2013, 97, 253-261. 
27. von Alvensleben, N.; Stookey, K.; Magnusson, M.; Heimann, K. Salinity tolerance of Picochlorum atomus and the use of salinity for contamination control by the freshwater cyanobacterium Pseudanabaena limnetica. PLoS ONE 2013, 8, e63569.

28. Davoli, P.; Mierau, V.; Weber, R.W.S. Carotenoids and fatty acids in red yeasts Sporobolomyces roseus and Rhodotorula glutinis. Appl. Biochem. Microbiol. 2004, 40, 392-397.

29. Easterling, E.R.; French, W.T.; Hernandez, R.; Licha, M. The effect of glycerol as a sole and secondary substrate on the growth and fatty acid composition of Rhodotorula glutinis. Bioresource Technol. 2009, 100, 356-361.

30. Islam, M.A.; Magnusson, M.; Brown, R.J.; Ayoko, G.A.; Nabi, M.N.; Heimann, K. Microalgal species selection for biodiesel production based on fuel properties derived from fatty acid profiles. Energies 2013, 6, 5676-5702.

31. Ramírez-Verduzco, L.F.; Rodríguez-Rodríguez, J.E.; Jaramillo-Jacob, A.R. Predicting cetane number, kinematic viscosity, density and higher heating value of biodiesel from its fatty acid methyl ester composition. Fuel 2012, 91, 102-111.

32. Roati, C.; Fiore, S.; Ruffino, B.; Marchese, F.; Novarino, D.; Zanetti, M.C. Preliminary evaluation of the potential biogas production of food-processing industrial wastes. Am. J. Environ. Sci. 2012, 8, 291-296.

33. Angelidaki, I.; Alves, M.; Bolzonella, D.; Borzacconi, L.; Campos, J.L.; Guwy, A.J. Defining the biomethane potential (BMP) of solid organic wastes and energy crops: A proposed protocol for batch assays. Water Sci. Technol. 2009, 59, 927-934.

34. Hidalgo, D. Evaluation of pre-treatment processes for increasing biodegradability of agro-food waste. Environ. Technol. 2012, 33, 1497-1503.

35. Velmurugan, B.; Ramanujam, R.A. Anaerobic digestion of vegetable wastes for biogas production in a fed-batch reactor. Int. J. Emerg. Sci. 2011, 1, 478-486.

36. Li, J.H.; Vasathan, T.; Rossnagel, B.; Hoover, R. Starch from hull-less barley: II. Thermal, rheological and acid hydrolysis characteristics. Food Chem. 2001, 74, 407-415.

37. Ariunbaatar, J.; Panico, A.; Esposito, G.; Pirozzi, F.; Lens, P.N.L. Pretreatment methods to enhance anaerobic digestion of organic solid waste. Appl. Energy 2014, 123, 143-156.

38. Li, Y.H.; Liu, B.; Sun, Y.; Zhao, Z.B.; Bai, F.W. Screening of oleaginous yeasts for broad-spectrum carbohydrates assimilating capacity. Chin. J. Biotechnol. 2005, 25, 39-43.

39. Sahu, A.; Pancha, I.; Jain, D.; Paliwal, C.; Ghosh, G.; Patidar, S.; Bhattacharya, S.; Mishra, S. Fatty acids as biomarkers of microalgae. Phytochemistry 2013, 89, 53-58.

40. Berner, F.; Heimann, K.; Sheehan, M. Microalgasl biofilms for biomass production. J. Appl. Phycol. 2014, doi:10.1007/s10811-014-0489-x.

41. Papanikolaou, S.; Aggelis, G. Lipids of oleaginous yeast. Part I. Biochemistry related with single cell oil production. Eur. J. Lipid Sci. Technol. 2011, 113, 1031-1051.

42. Xue, F.; Miao, J.; Zhang, X.; Luo, H.; Tan, T. Studies on lipid production by Rhodotorula glutinis fermentation using monosodium glutamate wastewater as culture medium. Bioresource Technol. 2008, 99, 5923-5927.

43. Schneider, T.; Graeff-Hönninger, S.; French, W.T.; Hernandez, R.; Merkt, N.; Claupein, W.; Hetrick, M.; Pham, P. Lipid and carotenoid production by oleaginous red yeast Rhodotorula glutinis cultivated on brewery effluents. Energy 2013, 61, 34-43. 
44. Dai, C.C.; Tao, J.; Xie, F.; Dai, Y.J.; Zhao, M. Biodiesel generation from oleaginous yeast Rhodotorula glutinis with xylose assimilating capacity. Afr. J. Biochem. Res. 2007, 6, 2130-2134.

45. Lin, L.; Zhou, C.; Vittayapadung, S.; Xiangqian, S.; Mingdong, D. Opportunities and challenges for biodiesel fuel. Appl. Energies 2011, 88, 1020-1031.

46. Yu, X.; Zheng, Y.; Dorgan, K.; Chen, S. Oil production by oleaginous yeasts using the hydrolysate from pretreatment of wheat straw with dilute sulfuric acid. Bioresource Technol. 2011, 102, 6134-6140.

47. Knothe, G. Fuel properties of highly polyunsaturated fatty acid methyl esters. Prediction of fuel properties of algal biodiesel. Energy Fuel 2012, 26, 5265-5273.

48. de Silva, M.J.; de Souza, S.N.M.; Chaves, L.I.; Rosa, H.A.; Secco, D.; Santos, R.F.; Barricatti, R.A.; Nogueira, C.E.C. Comparative analysis of engine generator performance using diesel oil and biodiesels available in Paraná State, Brazil. Renew. Sustain. Energy Rev. 2013, 17, 278-282.

49. Karthikeyan, O.P.; Visvanatha, C. Bio-energy recovery from high-solid organic substrates by dry anaerobic bio-conversion processes: A review. Rev. Environ. Sci. Bio/Technol. 2013, 12, 257-284.

50. Strong, P.J.; McDonald, B.; Gapes, D.J. Combined thermochemical and fermentative destruction of municipal biosolids: A comparison between thermal hydrolysis and wet oxidative pre-treatment. Bioresource Technol. 2010, 102, 5520-5527.

(C) 2015 by the authors; licensee MDPI, Basel, Switzerland. This article is an open access article distributed under the terms and conditions of the Creative Commons Attribution license (http://creativecommons.org/licenses/by/4.0/). 